PROCEEDINGS OF THE

AMERICAN MATHEMATICAL SOCIETY

Volume 129, Number 12, Pages 3569-3582

S 0002-9939(01)06034-8

Article electronically published on April 25, 2001

\title{
COMMUTATOR CONDITIONS IMPLYING THE CONVERGENCE OF THE LIE-TROTTER PRODUCTS
}

\author{
FRANZISKA KÜHNEMUND AND MARKUS WACKER
}

(Communicated by David R. Larson)

\begin{abstract}
In this paper we investigate commutator conditions for two strongly continuous semigroups $(T(t))_{t \geq 0}$ and $(S(t))_{t \geq 0}$ on a Banach space implying the convergence of the Lie-Trotter products $\left[T\left(\frac{\bar{t}}{n}\right) S\left(\frac{t}{n}\right)\right]^{n}$. The results are then applied to various examples and, in particular, to the Ornstein-Uhlenbeck operator.
\end{abstract}

\section{INTRODUCTION}

In 1959 H.F. Trotter [13] obtained an explicit product formula for semigroups whose generator is the sum of two generators. Using this idea, more general product formulas were considered by P.R. Chernoff [4, Ch. 1, 2]. Following this approach one can prove the theorem below (cf. [6, Ch. III, Cor. 5.8]).

Theorem 1. Let $(T(t))_{t \geq 0}$ and $(S(t))_{t \geq 0}$ be strongly continuous semigroups on a Banach space $E$ with generators $(A, D(A))$ and $(B, D(B))$, respectively, satisfying the stability condition

$$
\left\|\left[T\left(\frac{t}{n}\right) S\left(\frac{t}{n}\right)\right]^{n}\right\| \leq M e^{\omega t}
$$

for all $t \geq 0, n \in \mathbb{N}$, and some constants $M \geq 1, \omega \in \mathbb{R}$. Consider the sum $A+B$ on a subspace $D \subseteq D(A) \cap D(B)$ and assume that $D$ and $\left(\lambda_{0}-A-B\right) D$ are dense in $E$ for some $\lambda_{0}>\omega$.

Then the closure of $A+B$ exists and generates a strongly continuous semigroup $(U(t))_{t \geq 0}$ given by the Lie-Trotter product formula

$$
U(t) f=\lim _{n \rightarrow \infty}\left[T\left(\frac{t}{n}\right) S\left(\frac{t}{n}\right)\right]^{n} f
$$

where the limit exists for all $f \in E$ uniformly for $t$ in compact intervals in $\mathbb{R}_{+}$.

In [8] we gave an example of operators $A$ and $B$ such that the closure of the sum of $A$ and $B$ is a generator, but (1), and hence (2), is violated. Thus, to obtain the Lie-Trotter formula (2) a stability condition as in (1) is necessary.

On the other hand, in Theorem 1 one requires the range condition $\overline{\left(\lambda_{0}-A-B\right) D}$ $=E$, which is sometimes hard to verify. The aim of this work is to replace the

Received by the editors December 16, 1999 and, in revised form, April 14, 2000.

2000 Mathematics Subject Classification. Primary 34G10, 35K15, 47D06.

Key words and phrases. Strongly continuous semigroups, Trotter-Lie product formula, commutator conditions, Weyl relation, Ornstein-Uhlenbeck semigroup.

The authors thank Giorgio Metafune, Rainer Nagel, Abdelaziz Rhandi and Roland Schnaubelt for helpful discussions. 
range condition by assumptions on the commutator of $(T(t))_{t \geq 0}$ and $(S(t))_{t \geq 0}$, of $A$ and $B$, and their resolvents, respectively. At first glance, the commutator condition and introducing a new norm ||$|\cdot|||$ seems quite sophisticated. However, in the applications of our results in Section 4 and 5 we will see that we can use very simple norms. In particular, for the Ornstein-Uhlenbeck operator a natural Sobolev norm will work.

With our results we obtain a new approach for the Ornstein-Uhlenbeck semigroup in finite dimension as the limit of the Lie-Trotter products of a degenerate diffusion semigroup and a semigroup induced by a flow.

\section{Commutator CONDitions FOR SEMigroups AND GENERATORS}

To use commutator conditions to establish the convergence of the Lie-Trotter products, we modify the stability estimate (11).

Definition 2. Let $(T(t))_{t \geq 0}$ and $(S(t))_{t \geq 0}$ be semigroups on a normed vector space $(F,|| \cdot|| \mid)$. The semigroup $(T(t))_{t>0}$ is called exponentially bounded if

$$
\|T(t) \mid\| \leq M e^{\omega t}
$$

for all $t \geq 0$ and some constants $M \geq 1, \omega \in \mathbb{R}$.

The semigroups $(T(t))_{t \geq 0}$ and $(S(t))_{t \geq 0}$ are called locally Trotter-stable if there exist constants $t_{0}>0$ and $M_{t_{0}} \geq 1$ such that

$$
\text { || }\left[T\left(\frac{t}{n}\right) S\left(\frac{t}{n}\right)\right]^{n} \| \mid \leq M_{t_{0}}
$$

for all $t \in\left[0, t_{0}\right]$ and $n \in \mathbb{N}$.

With this concept, we are able to state our main result.

Theorem 3. Let $(T(t))_{t \geq 0}$ and $(S(t))_{t \geq 0}$ be strongly continuous semigroups on a Banach space $E$ and suppose that there exists a normed vector space $(F,|| \cdot|| \mid)$ which is densely embedded in $E$ and invariant under both semigroups such that the following conditions hold:

(a) The two semigroups are exponentially bounded on $F$ and locally Trotter-stable on $E$ and $F$.

(b) The commutator condition

$$
\|T(t) S(t) f-S(t) T(t) f\| \leq t^{\alpha} M_{1}\|\mid f\| \|
$$

is satisfied for all $f \in F$, and $t \in[0, \delta]$ with some $\delta>0$, and for some constants $\alpha>1$ and $M_{1} \geq 0$.

Then the Lie-Trotter product formula holds, i.e.,

$$
U(t) f:=\lim _{n \rightarrow \infty}\left[T\left(\frac{t}{n}\right) S\left(\frac{t}{n}\right)\right]^{n} f
$$

exists for all $f \in E$ uniformly for $t$ in compact intervals in $\mathbb{R}_{+}$. Moreover, $(U(t))_{t \geq 0}$ is a strongly continuous semigroup with generator $(G, D(G))$ such that

$$
G \supseteq \frac{d}{d t}[T(t) S(t)]_{\mid t=0} \supseteq A+B,
$$

where each operator is defined on its natural domain.

Proof. We denote the Lie-Trotter products by

$$
P_{n}(t):=\left[T\left(\frac{t}{n}\right) S\left(\frac{t}{n}\right)\right]^{n}
$$


for $n \in \mathbb{N}$ and $t \geq 0$. Let $t_{0}>0$ such that the stability condition (3) is fulfilled on $(E,\|\cdot\|)$ and $(F,\|\| \cdot \|)$. Therefore, we obtain

$$
\left\|P_{n}(t)\right\|=\left\|P_{k}\left(\frac{k t}{n}\right) P_{l}\left(\frac{l t}{n}\right)\right\| \leq M_{t_{0}}^{2}
$$

for $t \in\left[0, \frac{3 t_{0}}{2}\right]$ and $n=k+l, k, l \in \mathbb{N}$, such that $\frac{k t}{n}, \frac{l t}{n} \leq t_{0}, n \geq 2$. The analogous estimate also holds for \|\|$\cdot \|$. By induction we conclude that

$$
\left\|P_{n}(t)\right\| \leq M_{T} \quad \text { and } \quad\left\|P_{n}(t)\right\| \leq M_{T}
$$

for $(n, t) \in \mathbb{N} \times[0, T], T>0$, and a constant $M_{T} \geq 0$.

Now fix $T>0$ and let $t \in[0, T]$ and $f \in F$. We choose $k, m, n \in \mathbb{N}$ with $0<k \leq m$ such that $\frac{T}{m} \leq \delta$ and $m=k n$. Then, we obtain from the commutator condition (b) that

$$
\begin{aligned}
& \left\|T\left(\frac{t}{m}\right) S\left(\frac{j t}{m}\right) f-S\left(\frac{j t}{m}\right) T\left(\frac{t}{m}\right) f\right\| \\
& \quad \leq \sum_{l=0}^{j-1}\left\|S\left(\frac{l t}{m}\right)\left(T\left(\frac{t}{m}\right) S\left(\frac{t}{m}\right)-S\left(\frac{t}{m}\right) T\left(\frac{t}{m}\right)\right) S\left(\frac{(j-1-l) t}{m}\right) f\right\| \\
& \quad \leq j\left(\frac{t}{m}\right)^{\alpha} M_{2}\|\| f\|\|
\end{aligned}
$$

for $j \in \mathbb{N}$ and some constant $M_{2} \geq 0$. Hence, by forming a telescope sum, we have

$$
\begin{aligned}
& \left\|\left[T\left(\frac{k t}{m}\right) S\left(\frac{k t}{m}\right)\right] f-\left[T\left(\frac{t}{m}\right) S\left(\frac{t}{m}\right)\right]^{k} f\right\| \\
& \quad=\sum_{j=1}^{k-1}\left\|T\left(\frac{t j}{m}\right)\left(T\left(\frac{t}{m}\right) S\left(\frac{t j}{m}\right)-S\left(\frac{t j}{m}\right) T\left(\frac{t}{m}\right)\right) S\left(\frac{t}{m}\right)\left[T\left(\frac{t}{m}\right) S\left(\frac{t}{m}\right)\right]^{k-1-j} f\right\| \\
& \quad \leq M_{3} \sum_{j=1}^{k-1} j \cdot\left(\frac{t}{m}\right)^{\alpha}\||f|\| \\
& \quad=M_{3} \frac{t^{\alpha}(k-1) k}{2 m^{\alpha}}\|f\| \|
\end{aligned}
$$

for a suitable constant $M_{3} \geq 0$. Note that

$$
\left.\||| T\left(\frac{t}{m}\right) S\left(\frac{t}{m}\right)\right]^{j}\|\|=\left\|\left[T\left(\frac{t j / m}{j}\right) S\left(\frac{t j / m}{j}\right)\right]^{j}\right\| \mid \leq M_{T}
$$

for $j \in \mathbb{N}$ and $1 \leq j \leq m$. We now conclude that

$$
\begin{aligned}
& \left\|P_{n}(t) f-P_{m}(t) f\right\|=\left\|\left[T\left(\frac{k t}{m}\right) S\left(\frac{k t}{m}\right)\right]^{n} f-\left[T\left(\frac{t}{m}\right) S\left(\frac{t}{m}\right)\right]^{k \cdot n} f\right\| \\
& \leq \sum_{l=0}^{n-1}\left\|\left[T\left(\frac{k t}{m}\right) S\left(\frac{k t}{m}\right)\right]^{l}\left(T\left(\frac{k t}{m}\right) S\left(\frac{k t}{m}\right)-\left[T\left(\frac{t}{m}\right) S\left(\frac{t}{m}\right)\right]^{k}\right)\left[T\left(\frac{t}{m}\right) S\left(\frac{t}{m}\right)\right]^{k \cdot(n-1-l)} f\right\| \\
& \leq M_{4} \frac{t^{\alpha} n(k-1) k}{2 m^{\alpha}}\|f \mid\| \\
& \quad \leq M_{4} \frac{t^{\alpha} k}{2 m^{\alpha-1}}\|f\| \mid \\
& \leq M_{5} \frac{k}{2 m^{\alpha-1}}\|f\|
\end{aligned}
$$

for suitable constants $M_{4}, M_{5} \geq 0$. 
Thus, for arbitrary $i, j \in \mathbb{N}$ and $0 \leq t \leq T$, we have

$$
\begin{aligned}
\left\|P_{2^{i}}(t) f-P_{2^{j}}(t) f\right\| & \leq \sum_{l=i}^{j-1}\left\|P_{2^{l}}(t) f-P_{2^{l+1}}(t) f\right\| \\
& \leq M_{5} \sum_{l=i}^{j-1}\left(\frac{1}{2^{\alpha-1}}\right)^{l+1}\|\| f\|\| .
\end{aligned}
$$

The last expression converges to 0 as $i, j \rightarrow \infty$, since $\alpha>1$. Hence,

$$
U(t) f:=\lim _{i \rightarrow \infty} P_{2^{i}}(t) f
$$

exists for $f \in F$ uniformly for $t \in[0, T]$. Due to (44) we can extend $U(t)$ to a bounded linear operator on $E$.

For arbitrary $n \in \mathbb{N}$, we obtain from (5) that

$$
\begin{aligned}
\left\|P_{n}(t) f-U(t) f\right\| & \leq\left\|P_{n}(t) f-P_{n 2^{l}}(t) f\right\|+\left\|P_{n 2^{l}}(t) f-P_{2^{l}}(t) f\right\|+\left\|P_{2^{l}}(t) f-U(t) f\right\| \\
& \leq \sum_{k=0}^{l-1}\left\|P_{n 2^{k}}(t) f-P_{n 2^{k+1}}(t) f\right\|+\left\|P_{n 2^{l}}(t) f-P_{2^{l}}(t) f\right\|+\left\|P_{2^{l}}(t) f-U(t) f\right\| \\
& \leq \frac{M_{5}}{n^{\alpha-1}} \sum_{k=0}^{l-1}\left(\frac{1}{2^{\alpha-1}}\right)^{k+1}\|\mid f\|\left\|+M_{5} \frac{n}{\left(n 2^{l}\right)^{\alpha-1}}\right\| f\|\|+\left\|P_{2^{l}}(t) f-U(t) f\right\| \\
& \leq\left(\frac{2^{\alpha-1} M_{5}}{\left(2^{\alpha-1}-1\right) n^{\alpha-1}}+\frac{M_{5} n^{2-\alpha}}{2^{l(\alpha-1)}}\right)\||| f \mid\|+\left\|P_{2^{l}}(t) f-U(t) f\right\|
\end{aligned}
$$

for $l \in \mathbb{N}$.

Fix $\epsilon>0$. By the definition of the operators $U(t)$, there exists $l_{0} \in \mathbb{N}$ such that

$$
\left\|P_{2^{l}}(t) f-U(t) f\right\| \leq \epsilon
$$

for $f \in F$ and $l \geq l_{0}$. Further, for $n \in \mathbb{N}$ with $\left(\frac{1}{n}\right)^{\alpha-1} \leq \frac{\epsilon\left(2^{\alpha-1}-1\right)}{2^{\alpha-1} M_{5}}$ and $l \geq$ $\max \left\{l_{0}, l_{1}\right\}$ such that $2^{l_{1}(\alpha-1)} \geq\left(\frac{M_{5} n^{2-\alpha}}{\epsilon}\right)$, we have

$$
\begin{aligned}
\left\|P_{n}(t) f-U(t) f\right\| & \leq\left(\frac{2^{\alpha-1} M_{5}}{\left(2^{\alpha-1}-1\right) n^{\alpha-1}}+\frac{M_{5} n^{2-\alpha}}{2^{l(\alpha-1)}}\right)\|\| f\|+\| P_{2^{l}}(t) f-U(t) f \| \\
& \leq 3 \epsilon(\|f\|+\|f\| \|)
\end{aligned}
$$

for $f \in F$ uniformly for $t \in[0, T]$. So the stability condition (44) implies that $P_{n}(t)$ converges strongly in $E$ uniformly for $t \in[0, T]$.

To show the semigroup law for $(U(t))_{t \geq 0}$ and for rational numbers $t$, we use an idea from the paper of Chernoff (cf. [4, Thm. 2.5.1]). To that purpose, let $f \in E$ 
and $\epsilon>0$. Then

$$
\begin{aligned}
\| U(t) f- & U\left(\frac{t}{2}\right) U\left(\frac{t}{2}\right) f \| \\
& \leq\left\|U(t) f-\left[T\left(\frac{t}{2 n}\right) S\left(\frac{t}{2 n}\right)\right]^{2 n} f\right\|+\left\|\left[T\left(\frac{t}{2 n}\right) S\left(\frac{t}{2 n}\right)\right]^{2 n} f-U\left(\frac{t}{2}\right) U\left(\frac{t}{2}\right) f\right\| \\
& \leq \epsilon+\left\|\left[T\left(\frac{t}{2 n}\right) S\left(\frac{t}{2 n}\right)\right]^{n}\left(\left[T\left(\frac{t}{2 n}\right) S\left(\frac{t}{2 n}\right)\right]^{n} f-U\left(\frac{t}{2}\right)\right) f\right\| \\
& \quad+\left\|\left(\left[T\left(\frac{t}{2 n}\right) S\left(\frac{t}{2 n}\right)\right]^{n}-U\left(\frac{t}{2}\right)\right) U\left(\frac{t}{2}\right) f\right\| \\
& \leq 3 \epsilon
\end{aligned}
$$

for $n$ sufficiently large. This proves that $U(t)=U\left(\frac{t}{2}\right) U\left(\frac{t}{2}\right)$. In an analogous way, one can show $U(n t)=U(t)^{n}$ for $n \in \mathbb{N}$, from which one obtains the semigroup law for rational numbers.

By the uniform convergence of the Lie-Trotter products on compact intervals, we obtain that $(U(t))_{t \geq 0}$ is a strongly continuous semigroup. The assertion concerning the generator $G$ follows from [4, Prop. 4.1].

In the following, we show how commutator conditions on the generators $A$ and $B$ imply condition (b) of Theorem 3 . These conditions can be verified in some interesting applications (see Section 4 and 5 .

Definition 4. Let $(A, D(A))$ and $(B, D(B))$ be linear operators on a Banach space E. The operator

$$
\begin{gathered}
C:=A B-B A \quad \text { with domain } \\
D(C):=D(A B) \cap D(B A)=\{f \in D(A) \cap D(B): B f \in D(A) \text { and } A f \in D(B)\}
\end{gathered}
$$

is called the commutator of $A$ and $B$.

In the following we denote by $\|\cdot\|_{C}$ the $\mathbf{C}-$ norm defined by

$$
\|f\|_{C}:=\|f\|+\|C f\|
$$

for all $f \in D(C)$.

The next lemma allows to relate the commutator of $(T(t))_{t \geq 0}$ and $(S(t))_{t \geq 0}$ to the commutator $C$.

Lemma 5. Let $(A, D(A))$ and $(B, D(B))$ be generators of strongly continuous semigroups $(T(t))_{t \geq 0}$ and $(S(t))_{t \geq 0}$ on a Banach space $E$, and let $(C, D(C))$ be the commutator of $A$ and $B$. Suppose that there exists a subspace $F \subseteq D(C)$ which is dense in $E$ and invariant under $(T(t))_{t \geq 0}$ and $(S(t))_{t \geq 0}$ such that for some $\delta>0$ and $t \in[0, \delta]$ the functions

$$
\left\{\begin{array}{l}
s \mapsto A S(t) T(s) f, s \mapsto C S(s) f \text { are continuous and } \\
s \mapsto A S(s) f \text { is differentiable }
\end{array}\right.
$$

in $E$ for $s \in[0, \delta]$ and for all $f \in F$. Then the identity

$$
T(t) S(t) f-S(t) T(t) f=\int_{0}^{t} T(s)\left(\int_{0}^{t} S(t-r) C S(r) T(t-s) f d r\right) d s
$$

holds for all $f \in F$ and $t \in[0, \delta]$.

Proof. Let $t \in[0, \delta]$ with $\delta>0$. We apply the fundamental theorem of calculus to the continuously differentiable functions

$$
s \mapsto T(s) S(t) T(t-s) f \quad \text { and } \quad r \mapsto S(t-r) A S(r) f
$$


for $0 \leq r, s \leq \delta$ and $f \in F$. Since the operator $A$ is closed, we obtain by the differentiability of the second function that

$$
\frac{d}{d r} S(t-r) A S(r) f=S(t-r) C S(r) f
$$

for $0 \leq r \leq \delta$ and $f \in F$. Thus, we have

$$
\begin{aligned}
T(t) S(t) f-S(t) T(t) f & =\int_{0}^{t} \frac{d}{d s}\{T(s) S(t) T(t-s) f\} d s \\
& =\int_{0}^{t} T(s)(A S(t)-S(t) A) T(t-s) f d s \\
& =\int_{0}^{t} T(s)\left(\int_{0}^{t} \frac{d}{d r}\{S(t-r) A S(r) T(t-s) f\} d r\right) d s \\
& =\int_{0}^{t} T(s)\left(\int_{0}^{t} S(t-r) C S(r) T(t-s) f d r\right) d s
\end{aligned}
$$

for $f \in F$ and $t \in[0, \delta]$.

As an easy consequence of Lemma 5 we obtain the following result.

Corollary 6. Let $(A, D(A))$ and $(B, D(B))$ be generators of strongly continuous semigroups $(T(t))_{t \geq 0}$ and $(S(t))_{t \geq 0}$ on a Banach space $E$ and let $(C, D(C))$ be the commutator of $A$ and $B$. Suppose that:

(a) there exists a normed vector space $(F,|||\cdot| \mid), F \subseteq D(C)$, which is dense in $E$ and invariant under both semigroups, and the norm $|\|\cdot|\||$ is finer than the C-norm $\|\cdot\|_{C}$,

(b) there exists $\delta>0$ such that the functions

$$
\left\{\begin{array}{l}
s \mapsto A S(t) T(s) f, s \mapsto C S(s) f \text { are continuous and } \\
s \mapsto A S(s) f \text { is differentiable }
\end{array}\right.
$$

in $E$ for $s, t \in[0, \delta]$ and $f \in F$, and

(c) the two semigroups are exponentially bounded on $F$ and locally Trotter-stable on $E$ and $F$.

Then the conclusion of Theorem 3 holds.

Proof. By the exponential boundedness of the semigroups and Lemma 5 we obtain the estimate

$$
\begin{aligned}
\|T(t) S(t) f-S(t) T(t) f\| & =\left\|\int_{0}^{t} T(s)\left(\int_{0}^{t} S(t-r) C S(r) T(t-s) f d r\right) d s\right\| \\
& \leq M t^{2} \sup _{0 \leq r, s \leq t}\|S(r) T(t-s) f\|_{C} \\
& \leq \tilde{M} t^{2}\|\| f\|\|
\end{aligned}
$$

for $f \in F, t \in[0, \delta]$ and some constants $M, \tilde{M} \geq 0$. Thus, assumption (b) of Theorem $[3$ is fulfilled. Since condition (a) of Theorem 3 was assumed explicitly, the assertion follows. 


\section{Commutator CONDitions FOR RESOlvent OPERATORS}

In the following we express the commutator condition on the semigroups $(T(t))_{t \geq 0}$ and $(S(t))_{t \geq 0}$ in Theorem 3 by a corresponding condition on the powers of the resolvent operators $R(\lambda, A)$ and $R(\mu, B)$, respectively. In special cases, only a commutator condition on $R(\lambda, A)$ and $R(\mu, B)$ will be necessary.

First, we state the following result on the asymptotic behaviour of the gamma function.

Lemma 7. The gamma function defined by

$$
\Gamma(z)=\int_{0}^{\infty} t^{z-1} e^{-t} d t \quad \text { for } \operatorname{Re} z>0
$$

satisfies

$$
\lim _{n \rightarrow \infty} n^{b-a} \frac{\Gamma(n+a)}{\Gamma(n+b)}=1 \quad \text { for } a, b \in \mathbb{R} .
$$

Proof. By Stirling's Formula we can conclude that

$$
\Gamma(x+1)=\sqrt{2 \pi} x^{x+1 / 2} e^{-x}(1+\phi(x))
$$

for $x \in \mathbb{R}$, where $\phi$ is a function converging to zero as $\frac{1}{x}$ (see [1, p. 652]). Therefore, we have

$$
n^{b-a} \frac{\Gamma(n+a)}{\Gamma(n+b)}=\left(\frac{n+a-1}{n+b-1}\right)^{n+a-1 / 2}\left(\frac{n}{n+b-1}\right)^{b-a} e^{b-a} \frac{1+\phi(n+a-1)}{1+\phi(n+b-1)}
$$

for $a, b \in \mathbb{R}$ which converges to 1 as $n \rightarrow \infty$.

In the sequel, we assume without loss of generality that $(T(t))_{t \geq 0}$ and $(S(t))_{t \geq 0}$ are bounded semigroups. Note that we now use a commutator condition on the semigroups which is slightly different from the one in Theorem 3

Theorem 8. Let $(T(t))_{t \geq 0}$ and $(S(t))_{t \geq 0}$ be bounded strongly continuous semigroups on a Banach space $E$ with generators $(A, D(A))$ and $(B, D(B))$, respectively, and suppose that there exists a normed vector space $(F,\||\cdot|\|)$ which is embedded in E. Furthermore, let $\alpha, \beta \geq 0$ and $M \geq 0$. Then the following statements are equivalent:

(i) The semigroups satisfy the commutator condition

$$
\|T(t) S(s) f-S(s) T(t) f\| \leq t^{\alpha} s^{\beta} M \mid\|f\| \|
$$

for all $f \in F$ and $s, t \geq 0$.

(ii) The resolvent operators satisfy the commutator condition

$$
\left\|R(\lambda, A)^{n} R(\mu, B)^{n} f-R(\mu, B)^{n} R(\lambda, A)^{n} f\right\| \leq \frac{\Gamma(n+\alpha) \Gamma(n+\beta)}{(n-1) !^{2} \lambda^{\alpha+n} \mu^{\beta+n}} M\|\| f\|\|
$$

for all $f \in F, \lambda, \mu>0$ and $n \in \mathbb{N}$. 
Proof. (i) $\Rightarrow$ (ii). Let $f \in F$ and $\lambda, \mu>0$. We use the Laplace representation of the resolvent (see [6, Ch. II, Thm. 1.10]) and obtain

$$
\begin{array}{rl}
\| R(\lambda, A)^{n} & R(\mu, B)^{n} f-R(\mu, B)^{n} R(\lambda, A)^{n} f \| \\
& \leq \frac{1}{(n-1) !^{2}} \int_{0}^{\infty} \int_{0}^{\infty} r^{n-1} s^{n-1} e^{-\lambda r} e^{-\mu s}\|T(r) S(s) f-S(s) T(r) f\| d s d r \\
& \leq \frac{M}{(n-1) !^{2}} \int_{0}^{\infty} \int_{0}^{\infty} r^{n-1+\alpha} e^{-\lambda r} s^{n-1+\beta} e^{-\mu s} d r d s\||f|\| \\
& =\frac{M}{(n-1) !^{2} \lambda^{\alpha+n} \mu^{\beta+n}} \int_{0}^{\infty} r^{n-1+\alpha} e^{-r} d r \int_{0}^{\infty} s^{n-1+\beta} e^{-s} d s\|f\| \| \\
& =\frac{\Gamma(n+\alpha) \Gamma(n+\beta)}{(n-1) !^{2} \lambda^{\alpha+n} \mu^{\beta+n}} M\||\|f \mid\|
\end{array}
$$

for all $n \in \mathbb{N}$.

(ii) $\Rightarrow$ (i). Recall that by the Post-Widder Inversion Formula

$$
T(t) f=\lim _{n \rightarrow \infty}\left[\frac{n}{t} R\left(\frac{n}{t}, A\right)\right]^{n} f
$$

for $f \in E$ uniformly for $t$ in compact intervals in $\mathbb{R}_{+}$([ㅁ, Ch. III, Cor. 5.5 ]). Let $f \in F$ and $s, t \geq 0$. Applying Lemma 7 we conclude that

$$
\begin{aligned}
\frac{n^{2 n}}{t^{n} s^{n}} & \left\|R\left(\frac{n}{t}, A\right)^{n} R\left(\frac{n}{s}, B\right)^{n} f-R\left(\frac{n}{s}, B\right)^{n} R\left(\frac{n}{t}, A\right)^{n} f\right\| \\
& \leq \frac{n^{2 n}}{t^{n} s^{n}} \frac{\Gamma(n+\alpha) \Gamma(n+\beta)}{(n-1) !^{2}\left(\frac{n}{t}\right)^{\alpha+n}\left(\frac{n}{s}\right)^{\beta+n}} M|\||f|\| \\
& =n^{-(\alpha+\beta)} t^{\alpha} s^{\beta} \frac{\Gamma(n+\alpha) \Gamma(n+\beta)}{\Gamma(n)^{2}} M \mid\|f\| \| \\
& \longrightarrow t^{\alpha} s^{\beta} M|\|f\||
\end{aligned}
$$

as $n \rightarrow \infty$ which implies (i).

We now discuss some cases where it is enough to impose a commutator condition on the resolvent operators and not on all their powers.

Proposition 9. Let $(T(t))_{t \geq 0}$ and $(S(t))_{t \geq 0}$ be bounded strongly continuous semigroups on a Banach space $E$ with generators $(A, D(A))$ and $(B, D(B))$, respectively. Let $\alpha, \beta \geq 0$ such that $\alpha+\beta \geq 2$. Then the following statements are equivalent:

(i) The semigroups satisfy the commutator condition

$$
\|T(t) S(s) f-S(s) T(t) f\| \leq t^{\alpha} s^{\beta} M_{1}\|f\|
$$

for all $f \in E, s, t \geq 0$, and a constant $M_{1} \geq 0$.

(ii) The resolvent operators satisfy the commutator condition

$$
\|R(\lambda, A) R(\mu, B) f-R(\mu, B) R(\lambda, A) f\| \leq \frac{M_{2}}{\lambda^{\alpha+1} \mu^{\beta+1}}\|f\|
$$

for all $f \in E, \lambda, \mu>0, n \in \mathbb{N}$, and a constant $M_{2} \geq 0$.

Moreover, if $\alpha+\beta>2$, then the semigroups $(T(t))_{t \geq 0}$ and $(S(t))_{t \geq 0}$ commute.

Proof. The implication (i) $\Rightarrow$ (ii) is proved analogously as in Theorem 8 .

(ii) $\Rightarrow$ (i). For simplicity, we define

$$
\mathcal{R}_{A}\left(\frac{n}{t}\right):=\frac{n}{t} R\left(\frac{n}{t}, A\right), \mathcal{R}_{B}\left(\frac{n}{t}\right):=\frac{n}{t} R\left(\frac{n}{t}, B\right)
$$


and the commutator

$$
\left[\mathcal{R}_{A}\left(\frac{n}{t}\right), \mathcal{R}_{B}\left(\frac{n}{t}\right)\right]:=\frac{n}{t} R\left(\frac{n}{t}, A\right) \frac{n}{t} R\left(\frac{n}{t}, B\right)-\frac{n}{t} R\left(\frac{n}{t}, B\right) \frac{n}{t} R\left(\frac{n}{t}, A\right)
$$

for $n \in \mathbb{N}$ and $t \geq 0$. Since the semigroups are bounded there exists a constant $M \geq 0$ such that

$$
\left\|\left(\mathcal{R}_{A}\left(\frac{n}{t}\right)\right)^{j}\right\|,\left\|\left(\mathcal{R}_{B}\left(\frac{n}{s}\right)\right)^{j}\right\| \leq M
$$

for all $j, n \in \mathbb{N}$ such that $1 \leq j \leq n$, and for all $t, s \geq 0$. By assumption, we have

$$
\left\|\left[\mathcal{R}_{A}\left(\frac{n}{t}\right), \mathcal{R}_{B}\left(\frac{n}{t}\right)\right]\right\| \leq M_{2}\left(\frac{t}{n}\right)^{\alpha+\beta}
$$

for all $n \in \mathbb{N}, t \geq 0$ and some constant $M_{2} \geq 0$. Let $f \in E$ and $t, s \geq 0$. Then

$$
\begin{aligned}
\| T(t) & S(s) f-S(s) T(t) f \| \\
& =\lim _{n \rightarrow \infty}\left\|\left(\mathcal{R}_{A}\left(\frac{n}{t}\right)\right)^{n}\left(\mathcal{R}_{B}\left(\frac{n}{s}\right)\right)^{n} f-\left(\mathcal{R}_{B}\left(\frac{n}{s}\right)\right)^{n}\left(\mathcal{R}_{A}\left(\frac{n}{t}\right)\right)^{n} f\right\|
\end{aligned}
$$

and

$$
\begin{aligned}
&\left(\mathcal{R}_{A}\left(\frac{n}{t}\right)\right)^{n}\left(\mathcal{R}_{B}\left(\frac{n}{s}\right)\right)^{n} f-\left(\mathcal{R}_{B}\left(\frac{n}{s}\right)\right)^{n}\left(\mathcal{R}_{A}\left(\frac{n}{t}\right)\right)^{n} f \\
&=\mathcal{R}_{A}\left(\frac{n}{t}\right) {\left[\left(\mathcal{R}_{A}\left(\frac{n}{t}\right)\right)^{n-1},\left(\mathcal{R}_{B}\left(\frac{n}{s}\right)\right)^{n-1}\right] \mathcal{R}_{B}\left(\frac{n}{s}\right) f } \\
&+ {\left[\mathcal{R}_{A}\left(\frac{n}{t}\right),\left(\mathcal{R}_{B}\left(\frac{n}{s}\right)\right)^{n-1}\right]\left(\mathcal{R}_{A}\left(\frac{n}{t}\right)\right)^{n-1} \mathcal{R}_{B}\left(\frac{n}{s}\right) f } \\
&+\left(\mathcal{R}_{B}\left(\frac{n}{s}\right)\right)^{n-1}\left[\left(\mathcal{R}_{A}\left(\frac{n}{t}\right)\right)^{n}, \mathcal{R}_{B}\left(\frac{n}{s}\right)\right] f
\end{aligned}
$$

for all $n \in \mathbb{N}$. Moreover, we can estimate

$$
\begin{aligned}
\left\|\left[\left(\mathcal{R}_{A}\left(\frac{n}{t}\right)\right)^{n},\left(\mathcal{R}_{B}\left(\frac{n}{s}\right)\right)\right] f\right\| & \leq \sum_{j=0}^{n-1}\left\|\left(\mathcal{R}_{A}\left(\frac{n}{t}\right)\right)^{n-1-j}\left[\mathcal{R}_{A}\left(\frac{n}{t}\right), \mathcal{R}_{B}\left(\frac{n}{s}\right)\right]\left(\mathcal{R}_{A}\left(\frac{n}{t}\right)\right)^{j} f\right\| \\
& \leq n M^{2} M_{2}\left(\frac{t}{n}\right)^{\alpha}\left(\frac{s}{n}\right)^{\beta}\|f\| \\
\left\|\left[\mathcal{R}_{A}\left(\frac{n}{t}\right),\left(\mathcal{R}_{B}\left(\frac{n}{s}\right)\right)^{n-1}\right] f\right\| & \leq(n-1) M^{2} M_{2}\left(\frac{t}{n}\right)^{\alpha}\left(\frac{s}{n}\right)^{\beta}\|f\|,
\end{aligned}
$$

and by induction

$$
\left\|\left[\left(\mathcal{R}_{A}\left(\frac{n}{t}\right)\right)^{j},\left(\mathcal{R}_{B}\left(\frac{n}{s}\right)\right)^{j}\right] f\right\| \leq j^{2} M^{2} M_{2}\left(\frac{t}{n}\right)^{\alpha}\left(\frac{s}{n}\right)^{\beta}\|f\|
$$

for each $j, n \in \mathbb{N}$ such that $1 \leq j \leq n$. Therefore, equation (10) yields

$$
\begin{aligned}
\| T(t) & S(s) f-S(s) T(t) f \| \\
& =\lim _{n \rightarrow \infty}\left\|\left(\mathcal{R}_{A}\left(\frac{n}{t}\right)\right)^{n}\left(\mathcal{R}_{B}\left(\frac{n}{s}\right)\right)^{n} f-\left(\mathcal{R}_{B}\left(\frac{n}{s}\right)\right)^{n}\left(\mathcal{R}_{A}\left(\frac{n}{t}\right)\right)^{n} f\right\| \\
& \leq \lim _{n \rightarrow \infty} \frac{n^{2} t^{\alpha} s^{\beta}}{n^{\alpha+\beta}} M^{2} M_{2}\|f\| \\
& \leq t^{\alpha} s^{\beta} M_{1}\|f\|
\end{aligned}
$$

for some constant $M_{1} \geq 0$.

Using the estimate (11), it follows that the semigroups commute if $\alpha+\beta>2$.

A similar commutator condition for the resolvent operators in the context of nonautonomous evolution equations was studied in [9]. 


\section{Applications to Semigroups from quantum mechanics}

First, we apply our results to groups arising in quantum mechanics. Let $(T(t))_{t \in \mathbb{R}}$ and $(S(t))_{t \in \mathbb{R}}$ be a pair of unitary groups on a complex Hilbert space $H$ satisfying the so-called Weyl relation (see [10, p. 274])

$$
T(t) S(s)=e^{i s t} S(s) T(t) \text { for all } s, t \in \mathbb{R} .
$$

These semigroups are evidently exponentially bounded and locally Trotter-stable on $H$. Moreover, condition (b) of Theorem 3 follows from the estimate

$$
\|T(t) S(t) f-S(t) T(t) f\|=\left\|\left(e^{i t^{2}}-1\right) S(t) T(t) f\right\| \leq t^{2} \sum_{k=1}^{\infty} \frac{\left(t^{2}\right)^{k-1}}{k !}\|f\| \leq t^{2} e^{t^{2}}\|f\|
$$

for $f \in H$ and $t \geq 0$. Therefore, the Lie-Trotter product formula holds.

Based on this relation, P.E.T. Jørgensen, R.T. Moore [7, Ch. 11] and H. Suzuki [12] treated generalized Weyl relations leading to the following result.

Corollary 10. Let $(T(t))_{t \geq 0},(S(t))_{t \geq 0}$ and $(V(t))_{t \geq 0}$ be strongly continuous semigroups on a Banach space $E$ with generators $(A, D(A)),(B, D(B))$ and $(C, D(C))$, respectively. Suppose that there exists a subspace $F \subseteq D(C)$ which is dense in $E$ and invariant under the semigroups $(T(t))_{t \geq 0}$ and $(S(t))_{t \geq 0}$ such that the following conditions hold:

(a) The semigroups $(T(t))_{t \geq 0}$ and $(S(t))_{t \geq 0}$ are exponentially bounded on $\left(F,\|\cdot\|_{C}\right)$ and locally Trotter-stable on both $E$ and $\left(F,\|\cdot\|_{C}\right)$.

(b) The generalized Weyl relation holds, i.e.

$$
T(t) S(t) f=V\left(t^{2}\right) S(t) T(t) f
$$

for all $f \in E$ and $t \geq 0$.

Then the conclusion of Theorem $\mathbf{3}$ holds.

Proof. Fix $\delta>0$. We can estimate the commutator of $(T(t))_{t \geq 0}$ and $(S(t))_{t \geq 0}$ as

$$
\begin{aligned}
\|T(t) S(t) f-S(t) T(t) f\| & =\left\|\left(V\left(t^{2}\right)-I d\right)[S(t) T(t)] f\right\| \\
& =\left\|\int_{0}^{t^{2}} V(s) C[S(t) T(t)] f d s\right\| \\
& \leq t^{2} M\|f\|_{C}
\end{aligned}
$$

for $f \in F, t \in[0, \delta]$ and some constant $M \geq 0$. So Theorem[implies the assertion.

\section{Applications to Ornstein-Uhlenbeck operators}

In this section we consider the (finite-dimensional) Ornstein-Uhlenbeck operator which has been studied e.g. in [5]. Let $E:=C_{0}\left(\mathbb{R}^{d}\right)$ or $L^{p}\left(\mathbb{R}^{d}\right), 1 \leq p<\infty$. For any symmetric, positive semi-definite matrix $A:=\left(a_{i j}\right)$ and a matrix $B:=\left(b_{i j}\right) \in$ $\mathcal{L}\left(\mathbb{R}^{d}\right)$, the Ornstein-Uhlenbeck operator is defined by

$$
[\mathcal{O} f](x):=\sum_{i, j=1}^{d} a_{i j} D_{i j} f(x)+\sum_{i, j=1}^{d} b_{i j} x_{j} D_{i} f(x)=\langle\nabla, A \nabla f(x)\rangle+\langle B x, \nabla f(x)\rangle
$$

for each $f \in \mathcal{S}\left(\mathbb{R}^{d}\right), x \in \mathbb{R}^{d}$, the Schwartz space of rapidly decreasing functions, $x \in \mathbb{R}^{d}$, and $\nabla:=\left(\frac{\partial}{\partial x_{1}}, \ldots, \frac{\partial}{\partial x_{d}}\right)$. Without loss of generality, we write $A$ as 
$\operatorname{diag}\left(a_{1}, \ldots, a_{d}\right)$ where $a_{k}>0$ for $1 \leq k \leq j, a_{j+1}=\cdots=a_{d}=0$ and define the operators $\mathcal{A}$ and $\mathcal{B}$ as the closure of

$$
\mathcal{A} f:=\langle\nabla, A \nabla f\rangle \quad \text { and } \quad[\mathcal{B} f](x):=\langle B x, \nabla f(x)\rangle
$$

for $f \in \mathcal{S}\left(\mathbb{R}^{d}\right)$ and $x \in \mathbb{R}^{d}$, respectively (cf. [14, Ch. II.6]). The operator $(\mathcal{A}, D(\mathcal{A})$ ) generates a strongly continuous semigroup $(\mathcal{T}(t))_{t \geq 0}$ given by

$$
\mathcal{T}(t) f(x)=\frac{1}{\left(4 \pi t \prod_{i=1}^{j} a_{i}\right)^{j / 2}} \int_{\mathbb{R}^{j}} e^{-\sum_{i=1}^{j} \frac{\left|x_{i}-s_{i}\right|^{2}}{4 a_{i} t}} f\left(s_{1}, \cdots, s_{j}, x_{j+1}, \cdots, x_{d}\right) d s_{1} \cdots d s_{j}
$$

for $t>0, x \in \mathbb{R}^{d}$, and $f \in E$ (see [3]). Furthermore, the operator $(\mathcal{B}, D(\mathcal{B})$ ) generates the strongly continuous semigroup $(\mathcal{S}(t))_{t \geq 0}$ given by

$$
\mathcal{S}(t) f(x)=f\left(e^{t B} x\right)
$$

for all $f \in E$ and $x \in \mathbb{R}^{d}$ (see [․ Ch. II, Sec. 3.28]).

These semigroups and their generators have the following useful properties.

Lemma 11. Let $(\mathcal{T}(t))_{t \geq 0},(\mathcal{S}(t))_{t \geq 0}$ and their generators $(\mathcal{A}, D(\mathcal{A}))$ and $(\mathcal{B}, D(\mathcal{B}))$ be as above. Then the following properties hold:

(a) The semigroups $(\mathcal{T}(t))_{t \geq 0}$ and $(\mathcal{S}(t))_{t \geq 0}$ are locally Trotter-stable on $E$.

(b) The commutator $\mathcal{C}$ of $(\mathcal{A}, D(\mathcal{A}))$ and $(\mathcal{B}, D(\mathcal{B}))$ is given by

$$
\mathcal{C} f:=\mathcal{A B} f-\mathcal{B} \mathcal{A} f=2\langle B A \nabla, \nabla f\rangle
$$

for all $f \in \mathcal{S}\left(\mathbb{R}^{d}\right)$.

(c) For $t \geq 0$ the functions

$$
\left\{\begin{array}{l}
s \mapsto \mathcal{A S}(t) \mathcal{T}(s) f, s \mapsto \mathcal{C S}(s) f \text { are continuous and } \\
s \mapsto \mathcal{A S}(s) f \text { is differentiable }
\end{array}\right.
$$

in $E$ for $s \geq 0$ and for all $f \in \mathcal{S}\left(\mathbb{R}^{d}\right)$.

(d) The operator $\langle B A \nabla, \nabla\rangle$ commutes with $(\mathcal{T}(t))_{t \geq 0}$ on $\mathcal{S}\left(\mathbb{R}^{d}\right)$ and

$$
\langle B A \nabla, \nabla \mathcal{S}(t) f\rangle=\mathcal{S}(t)\left\langle e^{t B} B A e^{t B^{\star}} \nabla, \nabla f\right\rangle
$$

for $f \in \mathcal{S}\left(\mathbb{R}^{d}\right)$, where $B^{\star}$ denotes the transpose of the matrix $B$.

Proof. Clearly, the Schwartz space $\mathcal{S}\left(\mathbb{R}^{d}\right)$ is invariant under both semigroups and both generators $\mathcal{A}$ and $\mathcal{B}$. Moreover, $(\mathcal{T}(t))_{t \geq 0}$ is a semigroup of contractions on $E$ and

$$
\|\mathcal{S}(t) f\|_{C_{0}} \leq\|f\|_{C_{0}},\|\mathcal{S}(t) f\|_{L^{p}} \leq e^{t \omega}\|f\|_{L^{p}}
$$

for $f \in E$ and $\omega:=\frac{\operatorname{tr}(B)}{p}$. It follows that the semigroups are locally Trotter-stable on $E$.

To determine the commutator of $(\mathcal{A}, D(\mathcal{A}))$ and $(\mathcal{B}, D(\mathcal{B}))$ on $\mathcal{S}\left(\mathbb{R}^{d}\right)$, we note that

$$
\frac{\partial}{\partial x_{k}}\langle B x, \nabla f(x)\rangle=\left\langle B e_{k}, \nabla f(x)\right\rangle+\left\langle B x, \frac{\partial}{\partial x_{k}} \nabla f(x)\right\rangle
$$

for $1 \leq k \leq d, f \in \mathcal{S}\left(\mathbb{R}^{d}\right), x \in \mathbb{R}^{d}$, and the $k$-th unit vector $e_{k}$. Hence, we have

$$
\frac{\partial^{2}}{\partial^{2} x_{k}}\langle B x, \nabla f(x)\rangle=2\left\langle B e_{k}, \frac{\partial}{\partial x_{k}} \nabla f(x)\right\rangle+\left\langle B x, \nabla \frac{\partial^{2}}{\partial x_{k}^{2}} f(x)\right\rangle
$$


for $f \in \mathcal{S}\left(\mathbb{R}^{d}\right)$ and $x \in \mathbb{R}^{d}$. Therefore, we obtain from identity (16) that

$$
\begin{aligned}
{[\mathcal{A B} f-\mathcal{B} \mathcal{A} f](x)=} & 2 \sum_{k=1}^{d}\left\langle B e_{k} a_{k} \frac{\partial}{\partial x_{k}}, \nabla f(x)\right\rangle+\sum_{k=1}^{d}\left\langle B x, a_{k} \frac{\partial^{2}}{\partial x_{k}^{2}} \nabla f(x)\right\rangle \\
& \quad-\left\langle B x, \nabla \sum_{k=1}^{d} a_{k} \frac{\partial^{2}}{\partial x_{k}^{2}} f(x)\right\rangle \\
= & 2\langle B A \nabla, \nabla f(x)\rangle
\end{aligned}
$$

for $f \in \mathcal{S}\left(\mathbb{R}^{d}\right)$ and $x \in \mathbb{R}^{d}$.

Clearly, the operators $\mathcal{A}$ and $\mathcal{B}$ are continuous for the usual topology on the space $\mathcal{S}\left(\mathbb{R}^{d}\right)$ induced by the family of seminorms

$$
p_{\alpha, n}=\sup _{x \in \mathbb{R}^{d}}\left|x^{n} D^{\alpha} f(x)\right| .
$$

By a straightforward computation, using [11, Thm. 7.2 and Thm. 7.4], we obtain that the statements in (c) are fulfilled for the usual topology on $\mathcal{S}\left(\mathbb{R}^{d}\right)$. Since this topology is finer than the topology on $E$, assertion (c) is proved.

Observe that $(\mathcal{T}(t))_{t \geq 0}$ is a convolution semigroup and that convolution commutes with the differential operators $\frac{\partial}{\partial x_{k}}(1 \leq k \leq d)$. Therefore, the commutator of $(\mathcal{A}, D(\mathcal{A}))$ and $(\mathcal{B}, D(\mathcal{B}))$ commutes with $(\mathcal{T}(t))_{t \geq 0}$. For the semigroup $(\mathcal{S}(t))_{t \geq 0}$ we have the following commutator relation

$$
\nabla \mathcal{S}(t) f=\sum_{k=1}^{d} \mathcal{S}(t)\left\langle e^{t B} e_{k}, \nabla f\right\rangle e_{k},
$$

and therefore

$$
\langle B A \nabla, \nabla \mathcal{S}(t) f\rangle=\mathcal{S}(t)\left\langle e^{t B} B A e^{t B^{\star}} \nabla, \nabla f\right\rangle
$$

for $f \in \mathcal{S}\left(\mathbb{R}^{d}\right)$.

We can now apply Corollary 6 The fact that the closure of $\mathcal{A}+\mathcal{B}$ is a generator seems to be known (cf. [5]).

Proposition 12. Let $(\mathcal{T}(t))_{t \geq 0},(\mathcal{S}(t))_{t \geq 0}$ be the strongly continuous semigroups on $E$ given by (14) and (15) generated by $(\mathcal{A}, D(\mathcal{A}))$ and $(\mathcal{B}, D(\mathcal{B}))$, respectively. Then the conclusion of Theorem 3 holds.

Proof. On $\mathcal{S}\left(\mathbb{R}^{d}\right)$ we define the norm

$$
\|\| f\|:=\| f\left\|+\sum_{1 \leq i, j \leq d}\right\| \frac{\partial^{2}}{\partial_{i} \partial_{j}} f \|
$$

for $f \in \mathcal{S}\left(\mathbb{R}^{d}\right)$. This norm is finer than the $\mathcal{C}$-norm $\|\cdot\|_{\mathcal{C}}$.

In the next step, we show the exponential boundedness of the semigroups $(\mathcal{T}(t))_{t \geq 0}$ and $(\mathcal{S}(t))_{t \geq 0}$ on $\left(\mathcal{S}\left(\mathbb{R}^{d}\right),\||\cdot|\| \mid\right)$. This is obvious for $(\mathcal{T}(t))_{t \geq 0}$ which is $\|\cdot\| \mid \|$-contractive on $\mathcal{S}\left(\mathbb{R}^{d}\right)$. On the other hand, we have

$$
\left\|\frac{\partial^{2}}{\partial_{i} \partial_{j}} \mathcal{S}(t) f\right\|=\left\|\mathcal{S}(t)\left\langle e^{t B} e_{i j} e^{t B^{\star}} \nabla, \nabla f\right\rangle\right\| \leq e^{2 t \tilde{\omega}}\|\mathcal{S}(t)\| \cdot\|\mid\| f \|
$$


where

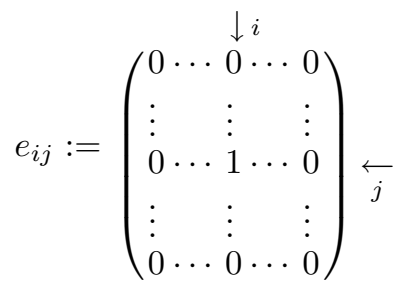

and $\tilde{\omega}:=\max _{1 \leq i, j \leq d}\left|b_{i j}\right|$. Therefore, we can estimate

$$
\||\mathcal{S}(t) f|\| \leq d^{2} e^{2 t \tilde{\omega}}\|\mathcal{S}(t)\| \cdot\|f|\||
$$

for $f \in \mathcal{S}\left(\mathbb{R}^{d}\right)$.

Finally, this implies the local Trotter-stability on $\left(\mathcal{S}\left(\mathbb{R}^{d}\right),|\|\cdot \mid\|)\right.$

$$
\left\|\left|\left[\mathcal{T}\left(\frac{t}{n}\right) \mathcal{S}\left(\frac{t}{n}\right)\right]^{n}\right|\right\| \leq e^{2 t \tilde{\omega}}|| f \mid \|
$$

if $E=C_{0}\left(\mathbb{R}^{d}\right)$, and

$$
\text { || }\left[\mathcal{T}\left(\frac{t}{n}\right) \mathcal{S}\left(\frac{t}{n}\right)\right]^{n}\left|\left\|\leq e^{t(\omega+2 \tilde{\omega})}|\|f \mid\|\right.\right.
$$

if $E=L^{p}\left(\mathbb{R}^{d}\right)$. We can now apply Corollary 6 which concludes the proof.

As a concrete example we mention a semigroup appearing in mathematical finance ([2]).

Examples 13. On the space $E:=C_{0}\left(\mathbb{R}^{2}\right)$ or $L^{p}\left(\mathbb{R}^{2}\right)(1 \leq p<\infty)$ the Cauchy problem

$$
\left\{\begin{aligned}
\frac{d}{d t} u(t, x, y) & =\frac{\partial^{2}}{\partial x^{2}} u(t, x, y)+x \frac{\partial}{\partial y} u(t, x, y), \quad t \geq 0, \\
u(0, x, y) & =f(x, y)
\end{aligned}\right.
$$

is investigated in [2]. Taking

$$
A=\left(\begin{array}{ll}
1 & 0 \\
0 & 0
\end{array}\right) \quad \text { and } B=\left(\begin{array}{ll}
0 & 0 \\
1 & 0
\end{array}\right)
$$

the Cauchy problem (17) can be reformulated as an Ornstein-Uhlenbeck operator $\mathcal{O}$ defined in (13). Therefore, all assumptions of Proposition 12 are satisfied and $\mathcal{O}$ generates a semigroup given as the limit of the Lie-Trotter products.

\section{REFERENCES}

[1] J. Bass, Cours de mathématiques. Tome I $4^{e}$ ed. Paris, Masson et $\mathrm{C}^{i e}$, Éditeurs, 1968. MR 37:1

[2] E. Barucci, S. Polidoro, V. Vespri, Some results on partial differential equations and Asian options, to appear.

[3] P. Cannarsa, G. Da Prato, On a functional approach to parabolic equations in infinite dimensions, J. Funct. Anal. 118(1) (1993), 22-42. MR 95a:35149

[4] P. R. Chernoff, Product formulas, nonlinear semigroups, and addition of unbounded operators, Mem. Amer. Math. Soc. 140 (1974), 1-121. MR 54:5899]

[5] G. Da Prato, A. Lunardi, On the Ornstein-Uhlenbeck operator in spaces of continuous fuctions, J. Funct. Anal. 131 (1995), 94-114. MR 97m:47056

[6] K.-J. Engel, R. Nagel, One-Parameter Semigroups for Linear Evolution Equations, Graduate Texts in Mathematics 194, Springer-Verlag, 2000. MR 2000i:47075

[7] P. E. T. Jørgensen, R. T. Moore, Operator Commutation Relations, Mathematics and its Application, D. Reidel Publishing Company, 1984. MR 86i:22006

[8] F. Kühnemund and M. Wacker, The Lie-Trotter product formula does not hold for arbitrary sums of generators, to appear in Semigroup Forum. 
[9] S. Monniaux and J. Prüss, A theorem of the Dore-Venni type for noncommuting operators, Trans. Amer. Math. Soc. 349(12) 1997, 4787-4814. MR 98b:47005

[10] M. Reed, B. Simon, Methods of Modern Mathematical Physics I. Functional Analysis, Academic Press, New York, 1972. MR 58:12429a

[11] W. Rudin, Functional Analysis, McGraw-Hill Book Company, 1973. MR 51:1315

[12] H. Suzuki, On the commutation relation $A B-B A=C$, Proc. Japan Acad. 47 (1971), 173-176. MR 45:966

[13] H. F. Trotter, On the product of semi-groups of operators, Proc. Amer. Math. Soc. 10 (1959), 545-551. MR 21:7446

[14] K. Yosida, Functional Analysis, Springer Verlag, 1974. MR 50:2851

Mathematisches Institut, Auf der Morgenstelle 10, D-72076 Tübingen, Germany

E-mail address: frku@michelangelo.mathematik.uni-tuebingen.de

Mathematisches Institut, Auf Der Morgenstelle 10, D-72076 Tübingen, Germany

E-mail address: mawa@michelangelo.mathematik.uni-tuebingen.de 